\title{
A Study on the Effect of Human Laterality, Type of Computer and Noise on Operators' Performance of a Data Entry Task
}

\author{
Zahid A. Khan \\ Sayed Aliul H. Rizvi \\ College of Engineering, King Abdulaziz University, Jeddah, Saudi Arabia
}

\begin{abstract}
Effects of human laterality, type of computer and noise on computer operators' performance of a data entry task were investigated. Seven right- and 7 left-motorsided male subjects performed the task on desktop and laptop computers in a sitting posture under varying noise levels. The mean number of characters entered per minute was statistically analysed. The right-motorsided operators entered more data than left-motorsided ones at each noise level. Operators performed better on desktop computers. Operators' performance decreased when noise level increased from 82 to $92 \mathrm{~dB}(\mathrm{~A})$ but it improved at $102 \mathrm{~dB}(\mathrm{~A})$. Effects of laterality, type of computer and noise, and the interaction between laterality and noise were statistically significant. The interactions between laterality and type of computer, and type of computer and noise were statistically insignificant. Laterality was statistically significant at all noise levels and noise was statistically significant for right motor-sided operators only.
\end{abstract}

equivalent noise level motor-sidedness visual display units data entry task performance

\section{INTRODUCTION}

With the rapidly increasing pace of computerization, the use of visual display units has been growing all over the world and, consequently, complaints by the end-users about their health impairment have also been pouring in at an alarming rate. Human problems associated with the human-computer interaction (HCI) environment need to be studied by researchers so that the users can use recent information and communication systems with comfort and with less adverse effects on their health. Studies on the capabilities and limitations of human performance through split brain research revealed that there were symmetrical behaviours in humans leading to a more efficient use of either the right or left parts of the body. This characteristic, referred to as human laterality or human motor-sidedness [1], has not been given due consideration in research in the area of HCI under the impact of noise-induced stress. Several studies showed health problems in keyboard operators connected with their jobs [2, 3, 4], e.g., noise negatively influenced human performance in tasks that require focused attention [5]. Visual cortical activity was shown to diminish substantially when subjects were exposed to loud sound [6]. Noise was also found to have a significantly negative effect on the performance of a readability task in a mobile driving environment [7]. However, several other studies showed no effect of noise on human psychomotor performance [5, 8, 9]. More work has to be carried out to be specific about noise effects. Keeping this in view, the present study was designed to investigate the effect of human laterality, type of computer and noise on operators' performance of a data entry task when the task was performed by the operators in a sitting posture at

Correspondence and requests for offprints should be sent to Zahid A. Khan, Industrial Engineering Department, College of Engineering, King Abdulaziz University, P.O. Box 80204, Jeddah - 21589, Saudi Arabia. E-mail: <zakhanusm@yahoo.com>. 
a desktop computer or a laptop under varying levels of noise. The following hypotheses were structured:

1. Human laterality (left or right motor-sidedness) has a significant effect on the operators' performance of the data entry task.

2. The type of computer (desktop or laptop) has a significant effect on the operators' performance of the data entry task.

3. The varying levels of noise significantly affect the operators' performance of the data entry task.

4. Interaction between human laterality and noise significantly affects the operators' performance of the data entry task.

5. Interaction between human laterality and the type of computer significantly affects the operators' performance of the data entry task.

6. Interaction between the type of computer and noise has a significant effect on the operators' performance of the data entry task.

7. Interaction between human laterality, the type of computer and noise has a significant effect on the operators' performance of the data entry task.

\section{METHOD}

\subsection{Subjects}

Seven right- and 7 left-motorsided male subjects participated in this study. The subjects' motorsidedness was determined on the basis of preference index $(P I)$ scores of Annet's inventory on motor-sidedness [10]. Subjects with $P I>.90$ were considered right-motorsided, whereas those with negative PI scores were accepted as leftmotorsided. Their age ranged from 18 to 22 years $(M=20.4, S D=2.6)$. All subjects had the same level of experience in computer operation and had normal vision. None had any history of neuromuscular disorders.

\subsection{Experimental Setup}

Experiments were conducted in an environmental chamber $4.9 \times 4.6 \times 2.9 \mathrm{~m}^{3}$. The temperature within the chamber was maintained at $\sim 25 \pm 2{ }^{\circ} \mathrm{C}$. When closed, the chamber was acoustically sealed from the outside environment. The level of illumination throughout the experimental session was maintained at $340 \mathrm{~lx}$ in accordance with International Labour Office's (ILO) recommendations [11] and was monitored with a TES 1330 digital light meter (TES EE Corp., Taiwan). The contrast ratio of the screen was 4:1 and was maintained throughout the experiment as recommended by ILO [11]. Screen luminance was $310 \mathrm{~cd} / \mathrm{m}^{2}$; the positions of the keyboard, monitor and documents were the same throughout the experimental session. The distance from the screen to the subjects' eyes was kept at $500 \mathrm{~mm}$ and the distance from the centre of the screen to the ground was $910 \mathrm{~mm}$ [12]. An audio-cassette recorder (Keltron India Ltd.) was used to play and replay pre-recorded noise. A GA-214 sound level meter (Castle Group of Industries, UK) was used to measure and monitor the noise level. A call bell was used to signal the beginning and the end of the experimental task. Finally, a digital watch to indicate the length of the experimental task (15 min), a Siva SM 1428 desktop computer (Sterling Computers Ltd., India) and an Armada 1350 laptop (Compaq, USA) were used in the study. The angle of the screen of both computers was kept at $110^{\circ}$, this being the most comfortable viewing position as suggested by the subjects. The text given to the subjects was in French in 12-pt regular Times New Roman, doublespaced. It was printed on a high quality white paper sheet. The sheet was in a document holder that remained fixed throughout the experimental session. The sheet contained words the subjects did not understand to minimize the difference between the subjects, whose level of proficiency in English was difficult to control.

\subsection{Experimental Procedure}

The following steps were taken before the actual experiment: (a) the subjects were briefed about the objectives of the experiment, (b) they were given instructions for the experimental task, and (c) they received sufficient training that familiarized them with the task. 
After a subject sat down in the chamber, the following steps were taken in both the training and experimental sessions: (a) a printed sheet that contained a text in French was presented to the subject, (b) a bell was triggered to signal the beginning of the experimental session, (c) the subject performed the data entry task for $15 \mathrm{~min}$ and task performance was measured in the mean number of characters entered per minute (MNCEPM), and (d) the bell was triggered to signal the end of the data entry task.

The experiments were conducted under varying levels of noise. The selected levels of noise, $L_{\text {eq }}$, were 82,92 and $102 \mathrm{~dB}(A)$ and they were played in a random order during the experimental sessions. When the task was performed, the level of noise was kept at a pre-specified value; the level was constantly monitored. The pre-specified levels of noise were maintained by manipulating the volume regulator of the audio-cassette player and they were measured near the subjects' ears. A 20-min rest period was provided before another set of experiments was carried out.

\subsection{Noise Levels}

A pilot study was carried out to determine the different levels of equivalent noise to which operators were subjected when working on computers in different work environments. For this purpose, sound levels at the New Delhi Railway Reservation office, Aashram Chowk, and the National Thermal Power Corporation (NTPC), Badarpur, in New Delhi, India, were recorded for 15 min on a Sony (Japan) audiocassette. They represented noise levels in office, traffic and factory environments respectively. The equivalent noise levels, $L_{\mathrm{eq}}$, in those locations were 82,92 and $102 \mathrm{~dB}(A)$, respectively.

\subsection{Statistical Analysis}

A 2 (motor-sidedness) $\times 2$ (type of computer) $\times$ 3 (levels of noise) analysis of variance (ANOVA) with repeated measures on the last two factors was used to determine the effects of the parameters under investigation. Human laterality (right and left motor-sidedness) and noise, $L_{\text {eq }}, 82,92$ and $100 \mathrm{~dB}(A)$, were the independent variables. Performance of a data entry task was the dependent variable; it was measured in MNCEPM. If the effect of noise was significant, a test for comparison among treatment means was undertaken to establish which means differed from one another. The trend analysis was also conducted to determine what kind of relationship existed between task performance (i.e., MNCEPM) and noise levels. Those analyses were related to the following four conditions of the experiment: condition I (when right motor-sided subjects performed a data entry task on a desktop computer at various noise levels), condition II (when right motor-sided subjects worked on a laptop under the impact of varying levels of noise), condition III (when left motor-sided subjects performed a data entry task on a desktop computer at various noise levels), and condition IV (when left motor-sided subjects worked on a laptop at varying levels of noise).

The comparison among treatment means for the four conditions was conducted using Newman Keul's test [13], whereas the trend analysis for those conditions was carried out using orthogonal polynomials [13].

\section{RESULTS}

Human performance, expressed in MNCEPM, is presented in Table 1: as the level of noise increased from 82 to $92 \mathrm{~dB}(A)$ both right and left motor-sided subjects entered fewer characters in a given period. However, MNCEPM increased with a further increase in noise level. Moreover, at each noise level, both right and left motorsided subjects entered more data on a desktop computer than on a laptop and performance of right motor-sided subjects was better than that of left motor-sided ones.

ANOVA revealed that the individual effects of human motor-sidedness, type of computer and noise were statistically significant (Table 2). Interaction between motor-sidedness and type of computer, type of computer and noise level, and also second-order interaction, i.e., motor-sidedness, type of computer and noise level, were found to be statistically insignificant. However, interaction between motor-sidedness and noise level emerged 
TABLE 1. Character Entry Rate at Different Noise Levels

\begin{tabular}{|c|c|c|c|c|c|c|}
\hline \multirow[b]{3}{*}{ Motor-Sidedness } & \multicolumn{6}{|c|}{ Character Entry Rate (MNCEPM) } \\
\hline & \multicolumn{3}{|c|}{ Desktop Computer } & \multicolumn{3}{|c|}{ Laptop } \\
\hline & $N_{1}$ & $\mathrm{~N}_{2}$ & $\mathbf{N}_{3}$ & $N_{1}$ & $\mathrm{~N}_{2}$ & $\mathbf{N}_{3}$ \\
\hline Right & 45.57 & 41.00 & 49.57 & 39.43 & 33.14 & 49.29 \\
\hline$(S D)$ & $(18.30)$ & $(15.82)$ & $(15.37)$ & $(14.28)$ & $(12.24)$ & $(19.33)$ \\
\hline Left & 36.14 & 29.71 & 34.43 & 24.43 & 20.29 & 24.86 \\
\hline$(S D)$ & $(9.46)$ & (7.95) & $(7.96)$ & $(5.65)$ & $(5.15)$ & $(7.47)$ \\
\hline $\begin{array}{l}\text { Statistical significance of } \\
\text { differences between means }\end{array}$ & $n s$ & $n s$ & $p \leq .05$ & $p \leq .05$ & $p \leq .05$ & $p \leq .05$ \\
\hline
\end{tabular}

Notes. MNCEPM—mean number of characters entered per minute; $N_{1}-82 \mathrm{~dB}(A), N_{2}-92 \mathrm{~dB}(A), N_{3}-102 \mathrm{~dB}(A)$.

TABLE 2. Results of an Analysis of Variance of the Character Entry Rate (MNCEPM) on a Desktop Computer and on a Laptop at Varying Noise Levels

\begin{tabular}{lcccc}
\hline Source of Variation & $\boldsymbol{d f}$ & $\boldsymbol{M S}$ & $\boldsymbol{F}$ & $\boldsymbol{p}$ \\
\hline Between subjects & 13 & 1116.14 & 1.34 & .3095 \\
Motor-sidedness & 1 & 4532.01 & 5.45 & .0378 \\
Subject within groups (error I) & 12 & 831.48 & 1.00 & .5000 \\
Within subjects & 70 & 58.07 & 1.39 & .2723 \\
Type of computer & 1 & 1181.25 & 28.40 & .0002 \\
Motor-sidedness $\times$ type of computer & 1 & 157.44 & 3.79 & .0753 \\
Type of computer $\times$ subject within group (error II) & 12 & 41.60 & 1.00 & .5000 \\
Noise & 2 & 517.19 & 29.23 & .0001 \\
Motor-sidedness $\times$ noise & 2 & 136.34 & 7.71 & .0026 \\
Noise $\times$ subject within group (error III) & 24 & 17.69 & 1.00 & .5000 \\
Type of computer $\times$ noise & 2 & 34.86 & 2.24 & .1282 \\
Motor-sidedness $\times$ type of computer $\times$ noise & 2 & 26.05 & 1.67 & .2094 \\
Type of computer $\times$ noise $\times$ subjects within group (error IV) & 24 & 15.58 & 1.00 & .5000 \\
\hline
\end{tabular}

Notes. MNCEPM-mean number of characters entered per minute.

TABLE 3. Results of a Comparison of the Character Entry Rate (MNCEPM) Obtained at Various Levels of Noise in 4 Experimental Conditions With Newman Keul's Test

\begin{tabular}{lcc}
\hline Conditions & Significant Difference Between & Nonsignificant Difference Between \\
\hline II & (i) $N_{2}$ and $N_{3}$ & (i) $N_{2}$ and $N_{1}$ \\
& (i) $N_{2}$ and $N_{1}$ & (ii) $N_{3}$ and $N_{1}$ \\
III & (ii) $N_{2}$ and $N_{3}$ & - \\
IV & (iii) $N_{1}$ and $N_{3}$ & $N_{3}$ and $N_{1}$ \\
& (i) $N_{2}$ and $N_{1}$ & $N_{3}$ and $N_{1}$ \\
& (ii) $N_{2}$ and $N_{3}$ & \\
\hline
\end{tabular}

Notes. MNCEPM-mean number of characters entered per minute; $N_{1}-82 \mathrm{~dB}(A), N_{2}-92 \mathrm{~dB}(A), N_{3}-102 \mathrm{~dB}(A)$.

TABLE 4. Results of Trend and Regression Analyses Between Noise Level and Character Entry Rate (MNCEPM)

\begin{tabular}{lcccc}
\hline Conditions & Significant Trend & Functional Relationship & $\boldsymbol{R}^{\mathbf{2}}$ & $\boldsymbol{r}$ \\
I & quadratic & $D=577.88-11.87 N+6.56 N^{2}$ & .998 & .997 \\
II & quadratic & $D=935.76-20.11 N+0.112 N^{2}$ & .998 & .997 \\
III & quadratic & $D=508.56-10.32 N+0.0556 N^{2}$ & .998 & .997 \\
IV & quadratic & $D=386.30-7.97 N+0.00434 N^{2}$ & .998 & .997 \\
\hline
\end{tabular}

Notes. $D$-mean number of characters entered per minute (MNCEPM), $N$-equivalent noise level in $\mathrm{dB}(A)$, $R^{2}$-coefficient of determination, $r$-coefficient of correlation. 
to be statistically significant (Table 2). A posthoc comparison of mean MNCEPM values obtained at various noise levels under the four experimental conditions is shown in Table 3. The results pertaining to trend analysis and nonlinear regression analysis are presented in Table 4.
Figure 1 shows the relationship between task performance and noise when right and left motor-sided operators entered data on a desktop computer. Similarly, Figure 2 shows the relationship between task performance and noise when right and left motor-sided operators entered

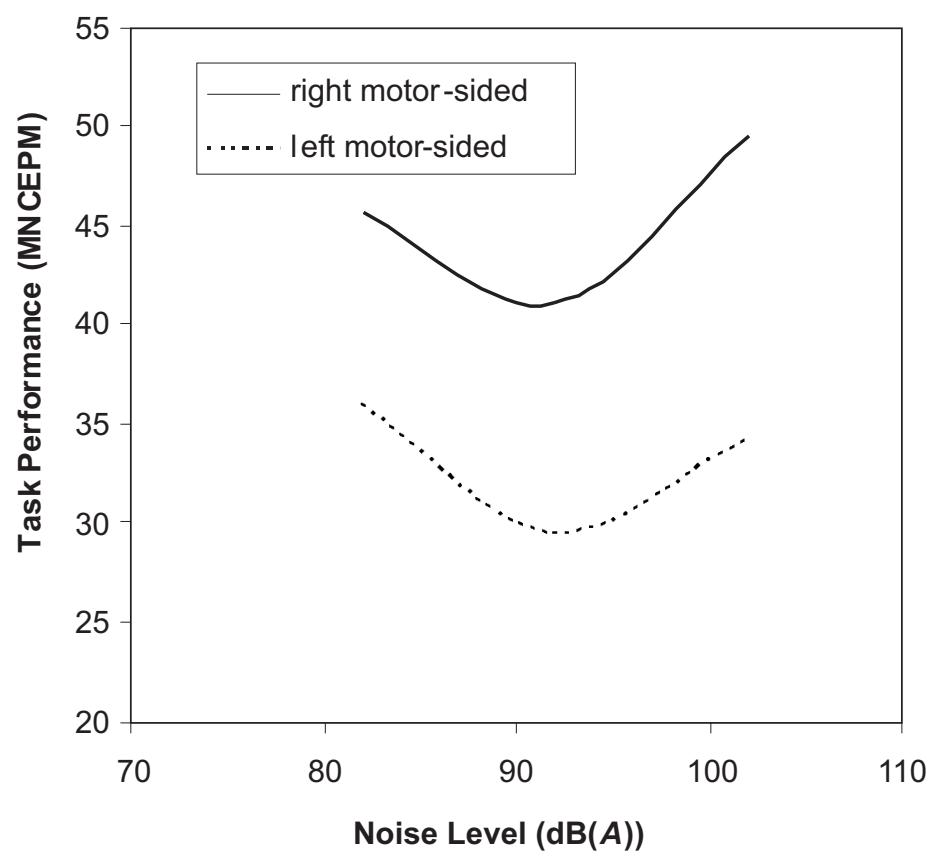

Figure 1. Relationship between task performance of right and left motor-sided operators on a desktop computer and noise level. Notes. MNCEPM—-mean number of characters entered per minute.

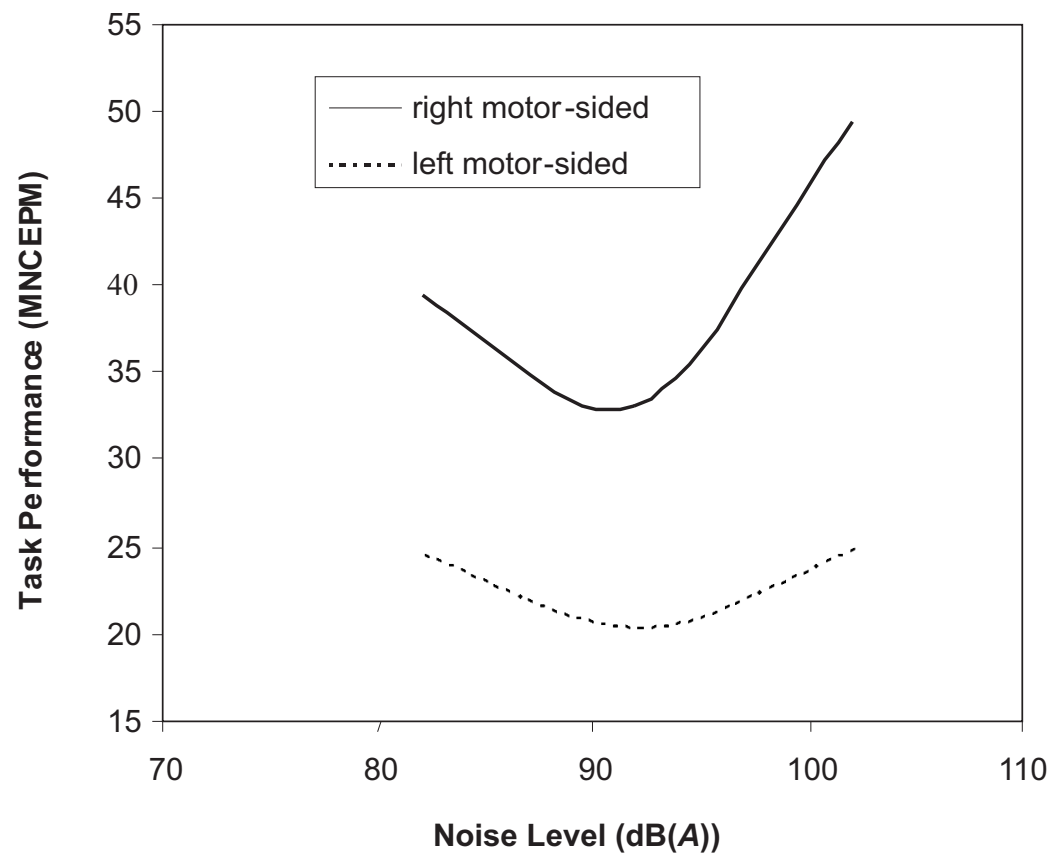

Figure 2. Relationship between task performance of right and left motor-sided operators on a laptop and noise level. Notes. MNCEPM-mean number of characters entered per minute. 
data on a laptop. An almost quadratic relationship can be seen in these figures.

With reference to ANOVA (Table 2) interaction between human motor-sidedness and noise turned out to be statistically significant. This significant interaction necessitated an analysis of simple main effects. Its results indicated that motor-sidedness was statistically significant at all three levels of noise and noise was statistically significant for right motor-sided operators only (Table 5).

TABLE 5. Analysis of the Simple Main Effect of the Subjects' Right and Left Motor-Sidedness at Varying Levels of Noise

\begin{tabular}{lccrc}
\hline Source of Variation & $\boldsymbol{d f}$ & $\boldsymbol{M S}$ & $\boldsymbol{F}$ & $\boldsymbol{p}$ \\
\hline Motor-sidedness at & & & & \\
$N_{1}$ & 1 & 1044.32 & 6.7 & .0117 \\
$N_{2}$ & 1 & 1020.03 & 6.5 & .0129 \\
$N_{3}$ & 1 & 2740.32 & 17.5 & .0001 \\
Noise level at & & & & \\
$\quad$ right motor-sidedness & 2 & 537.07 & 3.42 & .0381 \\
left motor-sidedness & 2 & 116.45 & 0.74 & .4807 \\
\hline
\end{tabular}

Notes. $N_{1}-82 \mathrm{~dB}(A), N_{2}-92 \mathrm{~dB}(A), N_{3}-102 \mathrm{~dB}(A)$.

\section{DISCUSSION}

ANOVA was used to test the hypotheses listed in section 1: the first four hypotheses were valid, whereas the last three were not. A literature review revealed few studies on the effect of laterality on human performance in the $\mathrm{HCI}$ environment. However, studies on a similar topic support the present findings. Daniels's [14] and Landaeur, Armstrong and Digwood's [15] studies on sensory motor co-ordination found significant differences in right and left motorsided people. Deluca, Sabbahi and Roy [16] reported that during sustained contractions the median frequency of integrated electromyogram signal decreased faster in the dominant hand of right-handed individuals, whereas no significant distinction could be found in left-handed subjects. They argued that such differences in righthanded subjects provided an indication of certain modifications in the metabolic properties of muscle fibres induced by a life time of preferred functional use. Hobday [3] showed that right- handed individuals differed from left-handed ones in keying performance. In another study, Badar [17] investigated the effect of laterality under varying levels of illumination in the $\mathrm{HCI}$ environment and concluded that human motorsidedness had a statistically significant effect on human performance.

Other studies also showed that laterality had a statistically significant effect on the performance of various tasks $[18,19]$. The findings of the present study suggested that in all the tasks that required co-ordination of two hands, laterality had a role to play. This point of view is further supported when it is observed that many persons are forced to use their nonpreferred hand in such skills as playing musical instruments, which requires fine motor control of both hands [20].

Another important finding based on the present study revealed that the type of computer significantly affected human performance. The study showed a higher relative efficiency in a task involving a desktop computer when compared with a laptop. The number of characters entered per minute when working on a laptop was much lower than when a desktop computer was used. This difference in performance might be traced in terms of the inherent design of the two kinds of computers. Because a desktop computer is bigger, operators might be more comfortable using the keyboard to enter data.

Another major finding of the present study pertains to the noise level that emerged to have a significant effect on human performance. The performance deteriorated with an increase in noise intensity to a certain level but improved with a further increase. This finding is supported by many studies which also reported that noise improved the performance of various tasks $[21,22,23]$. The long-term performance of a pilot under the impact of noise in the cockpit also improved [24]. This may be because lower levels of noise hindered performing the task and as the level of noise increased, the task became even more difficult. To execute it a person is more concentrated and applies extra effort; consequently, task performance is improved. 


\section{CONCLUSIONS}

On the basis of the results of this study (see section 4), the following conclusions were drawn.

- Right motor-sided computer operators achieved a higher rate of data entry than left motor-sided ones.

- The type of computer has a significant effect on data entry performance. Present research indicated that a desktop computer was more efficient for data entry task.

- Noise is a highly significant factor in affecting human performance in data entry in the HCI environment. Within the range considered in this study human performance deteriorated with an increase in noise intensity and then improved when that level was exceeded.

- The interaction between human laterality and noise was statistically significant.

- The interaction between human laterality and the type of computer was statistically insignificant for the data entry task considered in this study.

- The interaction between the type of computer and noise did not produce a significant effect on the operators' performance of a data entry task.

- The interaction between human laterality, the type of computer and noise was statistically insignificant for the data entry task considered in this study.

\section{REFERENCES}

1. Rizvi SAH. Effects of organismic and environmental factors on controls in a two wheeler driving configuration [doctoral dissertation]. Kanpur, India: Indian Institute of Technology Kanpur; 1984.

2. Life MA, Pheasant ST. An integrated approach to the study of posture in keyboard operation. Appl Ergon. 1984;15(2):83-90.

3. Hobday SW. Keyboard design to fit hands and reduce postural stress. In: Brown ID, Goldsmith R, Coombes K, Sinclair MA, editors. Proceedings of the 9th Congress of the International Ergonomics Association,
Bournemouth, UK. London, UK: Taylor \& Francis; 1985. p. 457-8.

4. Green DM, Fidell S. Variability in the criterion for reporting annoyance in community noise surveys. J Acoust Soc Am. 1991;89:234-43.

5. Smith AP. Noise and aspects of attention. Br J Psychol. 1991;82:313-24.

6. Cho ZH, Chung SC, Lim DW, Wong EK. Effects of the acoustic noise of the gradient system on fMRI: a study on auditory, motor, and visual cortices. Magn Reson Med. 1998;39:331-5.

7. Khan IA, Mallick Z, Khan ZA. A study on the combined effect of noise and vibration on operators' performance of a readability task in a mobile driving environment. International Journal of Occupational Safety and Ergonomics (JOSE). 2007;13(2):127-36.

8. Wheale JL, O'Shea NM. Noise and the performance of a four-choice psychomotor task. Ergonomics. 1982;25:1053-64.

9. Wilding J, Mohindra N, Breen-Lewis K. Noise effects in free recall with different orienting tasks. Br J Psychol. 1982;73:479-86.

10. Annet M. A classification of hand preference by association analysis. Br J Psychol. 1970;61:303-21.

11. International Labour Office (ILO). Working with visual display units (OSH series, No. 61). Geneva, Switzerland: ILO; 1989.

12. Grieco A, Molteni G, Piccoli B. Field study in news paper printing, a systematic approach to VDU operator strain. In: Grandjean E, Vigliani E, editors. Ergonomic aspects of VDTs. London, UK: Taylor \& Francis; 1980. p. 185-94.

13. Broota KD. Experimental design in behavioural research. New Delhi, India: Wiley Eastern; 1989.

14. Daniels FS. Do the eyes have it? American Rifleman. 1981;129:38-48.

15. Landaeur AA, Armstrong S, Digwood J. Sex differences in choice reaction time. $\mathrm{Br}$ J Psychol. 1980;71:551-5.

16. Deluca CJ, Sabbahi MA, Roy SH. 1986, Median frequency of the myoelectric signal. Effects of hand dominance. Eur $\mathrm{J}$ Appl Physiol. 1986;55:457-64. 
17. Badar MA. Studies on the effects of age and laterality under varying levels of illumination in an HCI environment [Master of Science thesis]. Aligarh, India: Aligarh Muslim University, Mechanical Engineering Department; 1990.

18. Cornell E, McManus IC. Differential survey response rates in right- and-left-handers. $\mathrm{Br}$ J Psychol. 1992;83:39-43.

19. Jone GV, Martin M. Handedness dependency in recall from everyday memory. $\mathrm{Br} \mathrm{J}$ Psychol. 1997;88:609-19.

20. Oldfield RC. Handedness in musicians. $\mathrm{Br}$ J Psychol. 1969;60:91-9.

21. Gawron VJ. Performance effects of noise intensity, psychological set, and task type and complexity. Hum Factors. 1982;24:225-43.

22. Hartley L, Dunne M, Schwartz S, Brown J. Effect of noise on cognitive strategies in a sentence verification task. Ergonomics. 1986;29:607-17.

23. Johansson CR. Effects of low intensity, continuous and intermittent noise on mental performance and writing pressure of children with different intelligence and personality characteristics. Ergonomics. 1983;26:275-88.

24. Stave AM. The effect of cockpit environment on long-term pilot performance. Hum Factors. 1977;19:503-14. 